Check for updates

Cite this: Chem. Commun., 2018, 54, 12021

Received 2nd August 2018, Accepted 17th August 2018

DOI: $10.1039 / \mathrm{c} 8 \mathrm{cc} 06304 d$

rsc.li/chemcomm

\section{The growth and fluorescence of phthalocyanine monolayers, thin films and multilayers on hexagonal boron nitride $\uparrow$}

\author{
Manal Alkhamisi, ${ }^{a}$ Vladimir V. Korolkov, ${ }^{a}$ Anton S. Nizovtsev, ${ }^{\text {bc }}$ James Kerfoot, ${ }^{a}$ \\ Takashi Taniguchi, ${ }^{d}$ Kenji Watanabe, (D) Nicholas A. Besley, ${ }^{\mathrm{b}}$ Elena Besley (D) ${ }^{\mathrm{b}}$ and \\ Peter H. Beton (D)*a
}

Free-base phthalocyanine forms distinct interfacial phases and thin films on hexagonal boron nitride including a monolayer arrangement as determined using high resolution atomic force microscopy. The phases reveal significant differences in photoluminescence with an intense peak for monolayer coverages of flat-lying molecules which is red-shifted in agreement with theoretical models.

The organisation of organic materials on a surface is important in realising their potential as electronic and optoelectronic devices, and is a major focus of supramolecular chemistry and materials science. ${ }^{1,2}$ In this study we investigate the influence of surface organisation on the fluorescence of free-base phthalocyanine $\left(\mathrm{H}_{2} \mathrm{Pc}\right.$; see inset in Fig. 1(b)). Phthalocyanines, both the free-base and metallated variants, have been widely exploited in organic electronics and gas-sensing, and also provide a prototypical example of an organic semiconductor on which many fundamental studies have been undertaken. ${ }^{3-12}$ Specifically we have investigated the adsorption of $\mathrm{H}_{2} \mathrm{Pc}$ on hexagonal boron nitride (hBN) and find that well-ordered monolayers can be formed in which the molecules lie flat with lattice vectors which are aligned with a high symmetry axis of the substrate, but additional layers grow in a three-dimensional morphology which is much less ordered. While the monolayer $\mathrm{H}_{2} \mathrm{Pc}$ gives rise to a narrow intense fluorescence peak the emission from the multilayers is much broader and significantly red-shifted.

$\mathrm{H}_{2} \mathrm{Pc}$ powder was purchased from Sigma-Aldrich and sublimed, under vacuum (base pressure $\sim 10^{-7} \mathrm{mbar}$ ), onto hBN

\footnotetext{
${ }^{a}$ School of Physics \& Astronomy, University of Nottingham, Nottingham NG7 2RD, UK. E-mail: peter.beton@nottingham.ac.uk

${ }^{b}$ School of Chemistry, University of Nottingham, Nottingham NG7 2RD, UK

${ }^{c}$ Nikolaev Institute of Inorganic Chemistry, Siberian Branch of the Russian Academy of Sciences, Academician Lavrentiev Avenue 3, 630090, Novosibirsk,

Russian Federation

${ }^{d}$ National Institute for Materials Science, 1-1 Namiki, Tsukuba, Ibaraki 305-0044, Japan

$\dagger$ Electronic supplementary information (ESI) available: Details of sample preparation; details of experimental techniques; details of calculations; additional images. See DOI: 10.1039/c8cc06304d
}
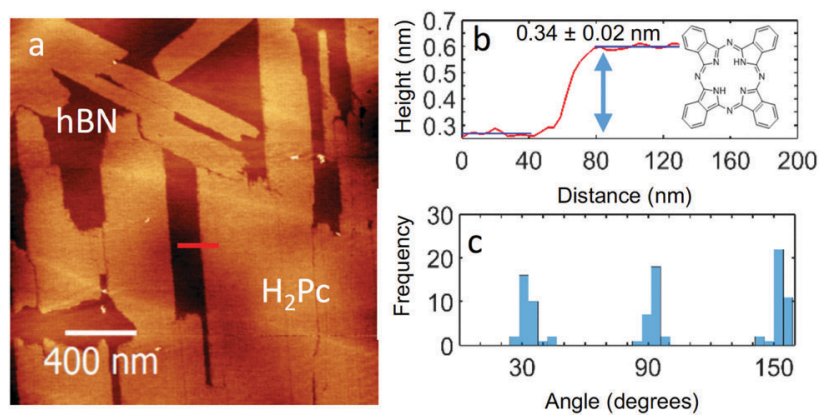

Fig. 1 (a) AC mode AFM image of $0.7 \mathrm{ML}$ of $\mathrm{H}_{2} \mathrm{Pc}$ on $\mathrm{hBN}$, (b) the height profile of an island along the red line marked in panel (a). (c) Histogram of island orientations.

flakes which had been exfoliated, transferred to a supporting $\mathrm{Si} / \mathrm{SiO}_{2}$ substrate and cleaned following the procedures summarised in ESI $\dagger$ and our recent work. ${ }^{13}$ The deposition rate was $\sim 0.5$ monolayers per min. $\mathrm{hBN}$ is chosen as a substrate since it is compatible with the formation of $2 \mathrm{D}$ supramolecular layers, and the acquisition of images with molecular resolution using atomic force microscopy (AFM) under ambient conditions. Since $\mathrm{hBN}$ is an insulator the fluorescence of the adsorbed molecular layers may also be measured, facilitating investigations of the correlation between morphology and optical properties. ${ }^{14,15}$

Fig. 1(a) shows the surface after 0.7 monolayer (ML) of $\mathrm{H}_{2} \mathrm{Pc}$ was deposited on hBN while the substrate was held at room temperature. Rectangular islands are observed with typical lengths ranging from 0.3 to $2 \mu \mathrm{m}$, and widths from 0.2 to $0.4 \mu \mathrm{m}$. The profile in Fig. 1(b) shows that the height of the islands is $0.34 \pm 0.02 \mathrm{~nm}$, consistent with the formation of a monolayer of $\mathrm{H}_{2} \mathrm{Pc}$ with the molecular plane lying parallel to the surface. The elongated island facets are oriented along specific directions - see histogram in Fig. 1(c) which has three peaks separated by $60^{\circ}$. This suggests that the major axes of the islands are aligned with a high symmetry axis of hBN.

If the substrate temperature is increased to $100{ }^{\circ} \mathrm{C}$, or higher, the growth self-terminates after the formation of a monolayer. 
The resulting islands are large (micron scale) and it is possible to acquire images with molecular scale resolution - see Fig. 2(a, $\mathrm{b}$ and d). The lattice vectors are marked in Fig. 2(b); we find that $a_{\mathrm{Pc}}=b_{\mathrm{Pc}}=2.18 \pm 0.05 \mathrm{~nm}$ and the angle $\theta=86^{\circ}$. The $\mathrm{H}_{2} \mathrm{Pc}$ molecules appear as topographically bright features with a 'crosslike' shape similar to the molecular structure (Fig. 1(b) inset). The orientation of the lattice vectors (red arrows) of the $\mathrm{H}_{2} \mathrm{Pc}$ monolayer and the hBN (blue arrows) can be determined by comparing AFM images of the $\mathrm{H}_{2} \mathrm{Pc}$ and the hBN lattice (see Fig. 2(c and d)). The lattice vectors of hBN may be resolved in Fig. 2(c) (parallel to the blue arrows) and are overlaid on the $\mathrm{H}_{2} \mathrm{Pc}$ molecules in Fig. 2(d). From these images we determine that the lattice vector $b_{\mathrm{Pc}}$ is aligned at angle of $30^{\circ}$ to both hBN lattice vectors, one of the high symmetry directions of the hBN substrate, consistent with the histogram in Fig. 1(c). The axis of the $\mathrm{H}_{2} \mathrm{Pc}$ molecules (parallel to the $\mathrm{C}-\mathrm{H}$ bonds within the central ring of the macrocycle) is misaligned from the hBN lattice vector by $4 \pm 2^{\circ}$.

A schematic of the $\mathrm{H}_{2} \mathrm{Pc}$ layer overlaid on $\mathrm{hBN}$ is shown Fig. 2(e). The molecules appear alternately bright (at the corners of the unit cell in Fig. 2(b and e)) and dark (at the centre of the unit cell). We suggest the reason for the differing AFM contrast of molecules is due to a variation in adsorption site; in our previous studies of molecular overlayers on hBN we have reported the appearance of moiré patterns ${ }^{13}$ in AFM images arising from a mismatch in lattice constants, and thus variations of local adsorption site. The $\mathrm{H}_{2} \mathrm{Pc}$ unit cell is similar to that previously reported on other substrates. ${ }^{16-19}$

We have calculated the adsorption energy and geometry of $\mathrm{H}_{2} \mathrm{Pc}$ using density functional theory (DFT). The structure of
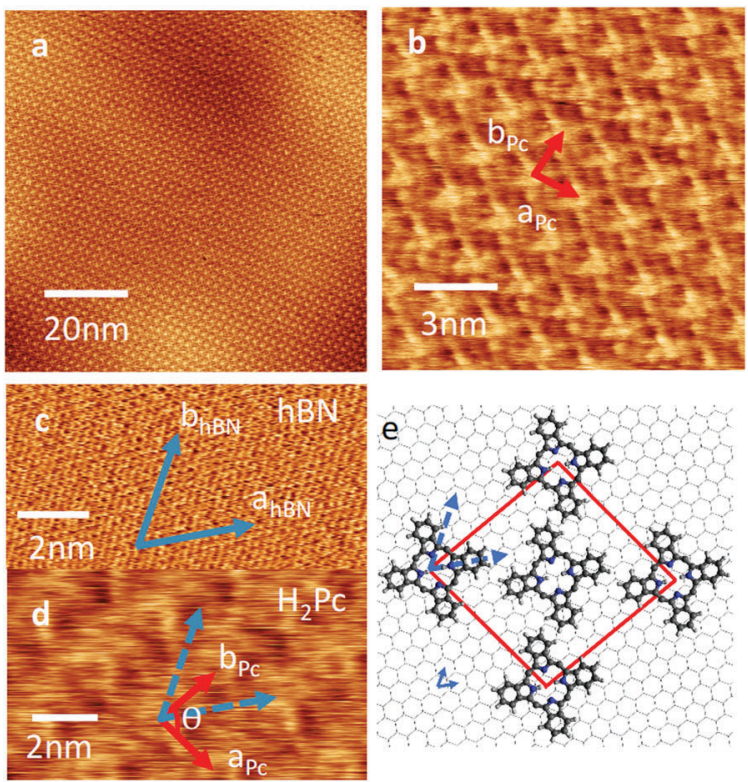

Fig. 2 ( $a$ and $b$ ) High resolution AFM images of a monolayer-thick island of $\mathrm{H}_{2} \mathrm{PC}$ grown at $300{ }^{\circ} \mathrm{C}$ showing the molecular arrangement on hBN. ( $c$ and d) AFM images used to determine the alignment of the molecular layer with the substrate. (e) Proposed schematic structure of $\mathrm{H}_{2} \mathrm{Pc}$ on hBN; the unit cell vectors of the molecular layer, $a_{\mathrm{Pc}_{\mathrm{c}}}$ and $b_{\mathrm{P}_{\mathrm{c}}}$, are marked by red arrows and subtend an angle $\theta$; the blue arrows denote the directions of the hBN lattice vectors.

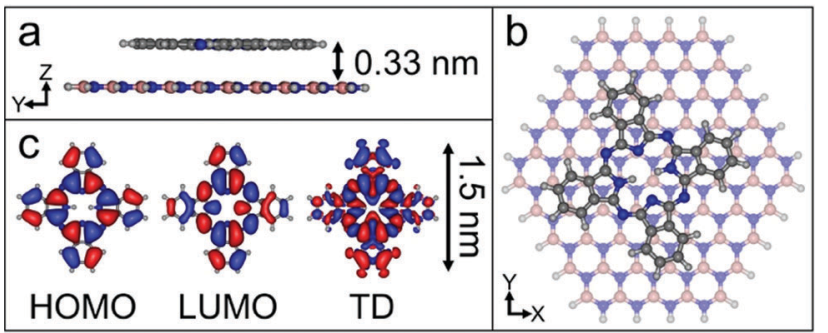

Fig. 3 The calculated adsorption site for $\mathrm{H}_{2} \mathrm{Pc}$ on hBN. (a) Cross-sectional view illustrating planarity of adsorption geometry and molecule-substrate separation. (b) Top view of $\mathrm{H}_{2} \mathrm{Pc}$ on hBN. (c) Highest occupied molecular orbital (HOMO, left) and lowest unoccupied molecular orbital (LUMO, centre) and transition density (TD, right) of $\mathrm{H}_{2} \mathrm{PC}$.

$\mathrm{H}_{2} \mathrm{Pc}$ on hBN is optimised using the range-separated hybrid $\omega \mathrm{B} 97 \mathrm{X}-\mathrm{D}$ functional, ${ }^{20}$ which includes an empirical dispersion correction, in combination with the correlation-consistent cc-pVDZ basis set. ${ }^{21}$ The atomic positions of a model hBN monolayer flake consisting of 65 boron atoms and 65 nitrogen atoms were initially optimized and frozen in subsequent calculations. The optimized structure for a single $\mathrm{H}_{2} \mathrm{Pc}$ molecule on hBN is shown in Fig. 3 and confirms a planar adsorption geometry with a molecule-surface separation, $d=0.33 \mathrm{~nm}$ and adsorption energy, $E_{\mathrm{A}}=3.35 \mathrm{eV}$. In common with our images the calculations show the molecular axis misaligned with the hBN lattice vector, but by a larger angle, $13^{\circ}$. We attribute this difference to a combination of a low barrier for rotation and the role of intermolecular interactions. Full details of the calculations, which are performed within the QCHEM package, ${ }^{22}$ are included in ESI. $\dagger$

As the $\mathrm{H}_{2} \mathrm{Pc}$ coverage is increased we observe the formation of clusters of large needle-like crystallites with a rather disordered morphology. Fig. 4(a) shows a large area AFM image of an hBN flake in which several large islands (identified by arrows) are formed following the deposition of $\mathrm{H}_{2} \mathrm{Pc}$ (thickness $1 \mathrm{~nm}$; $1 \mathrm{ML}$ is approximately equivalent to $0.33 \mathrm{~nm}$ ). These islands have a height of 20-30 nm (see Fig. 4(c)) and typical length of 10-20 $\mu \mathrm{m}$, and can be resolved using optical microscopy; Fig. 4(b) shows the same hBN flake. Interestingly the islands have a direction which is locally correlated and oriented in one of three directions subtended by $60^{\circ}$. Following the deposition of thicker layers (Fig. 4(d)) the needle-like islands merge to cover a greater proportion of the surface, but are highly disordered. We are not able to obtain images with molecular-scale resolution in these regions. Overall our results show an abrupt transition to the growth of three-dimensional crystallites following the completion of a highly ordered monolayer. A likely explanation for this is that molecules switch from the flat-lying adsorbed state in the monolayer to a geometry in which the molecular plane is at an angle to the hBN substrate in higher layers, thus forming one-dimensional crystallites in which molecules are co-planar, maximising $\pi-\pi$ interactions. Such a switch from a flat-lying to upright morphology has been observed previously in studies of adsorbed $\mathrm{H}_{2} \mathrm{Pc}^{4}$

To measure the optical properties of a $\mathrm{H}_{2} \mathrm{Pc}$ thin film, a Horiba LabRAM system was used in conjunction with a 

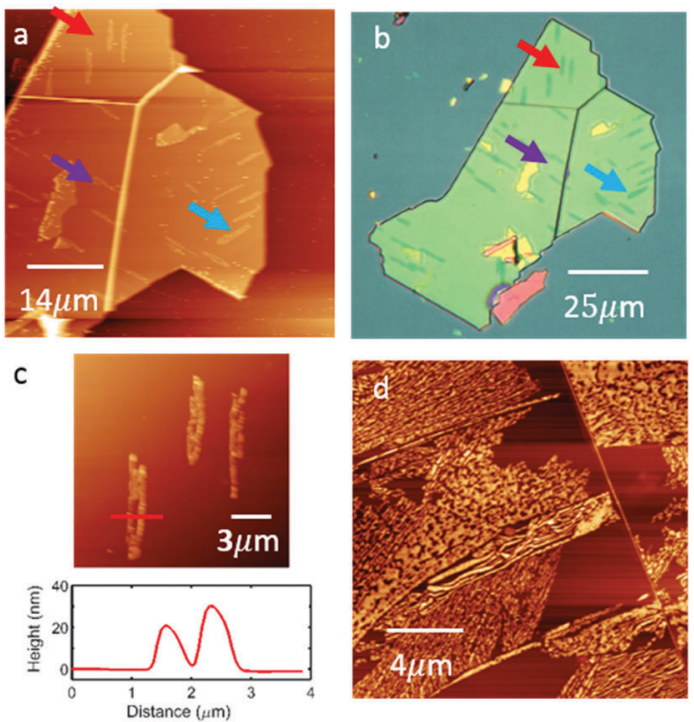

Fig. 4 (a) AC mode AFM image of $1 \mathrm{~nm}$ thin film of free-base phthalocyanine on hBN, (b) an optical micrograph of the same flake. Both images reveal needle-like multilayer crystallites and the arrows with same colours show the same islands in both optical and AFM images. (c) Profile of needle-line island showing typical island height in the range $20-30 \mathrm{~nm}$. (d) AC mode AFM of $32 \mathrm{~nm}$ thick film of free-base phthalocyanine on $\mathrm{hBN}$ at room temperature.

$660 \mathrm{~nm}$ laser (photon energy $1.88 \mathrm{eV}$ ) with a spot size $\sim 1 \mu \mathrm{m}^{2}$ as an excitation source (see ESI $\dagger$ for further details). In Fig. 5 the photoluminescence spectra of $\mathrm{H}_{2} \mathrm{Pc}$ films with varying thickness are shown. For a thickness of $0.7 \mathrm{ML}$, in which all molecules adsorb in a flat-lying configuration, we observe a narrow intense peak centred at $1.75 \mathrm{eV}$, which we attribute to the zero phonon (0-0) peak, together with broader, less intense vibronic peaks at 1.67 and $1.58 \mathrm{eV}$. These peaks are also present

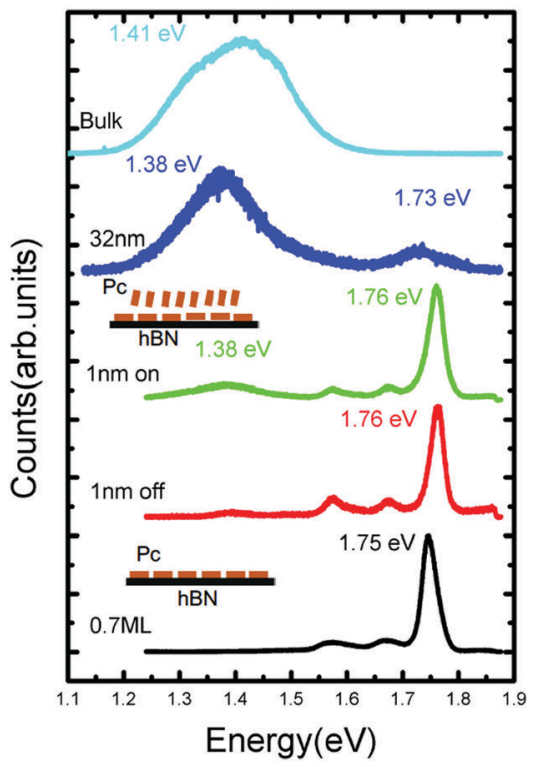

Fig. 5 The photoluminescence spectra of free-base phthalocyanine films with different thickness on and off the islands. for a sample with $\mathrm{H}_{2} \mathrm{Pc}$ thickness of $1 \mathrm{~nm}$ (as in Fig. 2), but undergo a small shift; the $0-0$ peak is centred on $1.76 \mathrm{eV}$. There are small differences between the spectra acquired when the laser spot is incident either on or off a multilayer $\mathrm{H}_{2} \mathrm{Pc}$ island. In particular, an additional peak centred at $1.38 \mathrm{eV}$ is slightly more intense on these islands. This peak is much more intense for a thicker sample (32 nm), for which the peaks associated with the flat-lying monolayer may no longer be clearly resolved. We have also measured the spectrum of $\mathrm{H}_{2} \mathrm{Pc}$ powder on a glass slide which showed a broad peak at $1.42 \mathrm{eV}$ (light blue line in Fig. 5) and we attribute the peak observed at $1.38 \mathrm{eV}$ for the $32 \mathrm{~nm}$ thick $\mathrm{H}_{2} \mathrm{Pc}$ layer to the existence of a bulk-like $\mathrm{H}_{2} \mathrm{Pc}$ phase on the surface.

The 0-0 peak for $\mathrm{H}_{2} \mathrm{Pc}$ is red-shifted by $\Delta E=0.13 \mathrm{eV}$ as compared with a molecule in the gas phase ${ }^{23}(0-0$ peak at $1.876 \mathrm{eV})$. As we, ${ }^{14,15}$ and others, ${ }^{24-26}$ have recently discussed this red-shift can be understood from a combination of effects arising from interactions with the substrate and with nearest neighbours. A non-resonant contribution to the shift due to the substrate can be calculated using time-dependent density functional theory (TD-DFT). The molecule is optimized in the excited $\left(\mathrm{S}_{1}\right)$ state both on (adsorbed) and off (gas phase) the hBN surface and the transition to the ground state $\left(S_{0}\right)$ is calculated in each case (see ESI $\dagger$ for more details). The calculated transition energies for a molecule on the hBN surface, and in the gas phase are, respectively, $1.76 \mathrm{eV}$ and $1.87 \mathrm{eV}$. These values are close to the measured peaks in fluorescence and their difference implies a non-resonant red-shift $\Delta E_{\mathrm{nr}}=0.11 \mathrm{eV}$ due to adsorption on hBN.

There are also contributions to the red-shift from resonant interactions with the substrate and neighbouring molecules. We have recently argued $^{15}$ that these effects depend on the refractive index, $n$, of the substrate and include a 'gas-surface' red-shift given by $\Delta E_{\text {subs }}=f(d)\left(n^{2}-1\right) \mu^{2} / 4 \pi \varepsilon_{0}\left(n^{2}+1\right) d^{3}$, where $\mu$ is the transition dipole moment and $d$ is the molecule-substrate separation, which from our DFT calculations (see ESI $\dagger$ ) are, respectively, 8.0 Debye and $0.33 \mathrm{~nm}$ (for the anisotropic hBN substrate we use a geometric average ${ }^{15} n=1.87$; the reduction factor $f(d)$ is related to the molecular geometry as discussed below). This shift arises from a coupling of the transition dipole moment of the adsorbed molecule with the dielectric hBN substrate and may be described semi-classically as an interaction with an image dipole (see Kerfoot et $a l .{ }^{15}$ ).

In the point dipole approximation $f(d)=1$; this is satisfied if $d$ is much greater than the molecular dimensions. However, this condition is violated for $\mathrm{H}_{2} \mathrm{Pc}$ (see dimensions in Fig. 3); instead we treat the dipole moment as arising from an oscillating charge density, ${ }^{27}$ the transition density, $T(r)$, equal to the product of the wave functions of the lowest unoccupied molecular orbital (LUMO) and the highest occupied molecular orbital (HOMO). Previously ${ }^{15}$ we have used the extended dipole model to determine an approximate expression for $f(d)$, however this is expected to be valid only for linear molecules. For the cross-shaped $\mathrm{H}_{2} \mathrm{Pc}$ molecule we instead calculate the reduction factor due to the interaction of the charge density described by $T(r)$ with its image, $T^{\prime}\left(r^{\prime}\right)$, given by 
$f(d)=A \iint \mathrm{d} r \mathrm{~d} r^{\prime} T(r) T\left(r^{\prime}\right) / 4 \pi \varepsilon_{0}\left|r-r^{\prime}+\mathrm{d} \hat{z}\right|$, where the constant $A=\mu^{2} / 4 \pi \varepsilon_{0} d^{3}$ and $T^{\prime}\left(x^{\prime}, y^{\prime}, z^{\prime}\right)=T\left(x^{\prime}, y^{\prime},-z^{\prime}\right)$. The isosurfaces of the HOMO, LUMO and the transition density are shown in Fig. 3. Evaluating numerically this double integral using the transition density calculated using DFT gives, for $d=0.33 \mathrm{~nm}$, $f(d)=0.106$ and $\Delta E_{\text {subs }}=0.03 \mathrm{eV}$.

We might also expect a red-shift due to a screened resonant interaction with neigbouring molecules; ${ }^{24}$ the characteristic energy for a pairwise interaction is $\Delta E_{\mathrm{nn}}=\mu^{2} / 2\left(n^{2}+1\right) \pi \varepsilon_{0} r_{\mathrm{nn}}{ }^{3}$, where $r_{\mathrm{nn}}=1.5 \mathrm{~nm}$, the nearest neighbour separation. We find $\Delta E_{\mathrm{nn}}=5 \mathrm{meV}$; this red-shift is expected for molecules which have aligned transition dipole moments. From our DFT calculations we find that the transition dipole moment for $\mathrm{H}_{2} \mathrm{Pc}$ is oriented close to perpendicular to the $\mathrm{C}-\mathrm{H}$ bonds within the central ring of the macrocycle. From our images we are unable to determine whether the molecules are aligned (i.e. whether the $\mathrm{C}-\mathrm{H}$ bonds within all molecules have a common alignment). However, the contrast of the islands, which may be resolved in optical microscopy, shows no dependence on polarisation indicating that the transition dipole moments are not ordered. Assuming a random alignment, the total red-shift due to $N$ nearest neighbours is $\sim \chi^{2} N \Delta E_{\mathrm{nn}} / 2$ where $\chi^{2}$ is the Franck Condon factor. ${ }^{24}$ Since $\chi^{2} \leq 1$ we estimate that this contribution to the gas-surface red-shift is $\sim 10 \mathrm{meV}$. Combining the various calculated contributions to the red-shift gives $\Delta E_{\mathrm{tot}}=\Delta E_{\mathrm{nr}}+\Delta E_{\text {subs }}+\Delta E_{\mathrm{nn}}$ in the range $0.14-0.15 \mathrm{eV}$ in excellent agreement with the experimental value, $0.13 \mathrm{eV}$.

Overall our results demonstrate that the optoelectronic properties of molecules on surfaces depends strongly on their adsorption geometry and environment as determined, with molecular resolution, using atomic force microscopy. The absorption of $\mathrm{H}_{2} \mathrm{Pc}$ in a flat lying geometry results in a narrow intense emission peak, but evolves to a broad emission feature for multilayer samples.

This work was supported by the Engineering and Physical Sciences Research Council [grant number EP/N033906/1]; the Leverhulme Trust [grant number RPG-2016-104]. Nottingham Nanoscale and Microscale Research Centre enabled access to the Horiba LABRAM instrument. K. W. and T. T. acknowledge support from the Elemental Strategy Initiative conducted by the MEXT, Japan and the CREST (JPMJCR15F3), JST. EB acknowledges the financial support of an ERC Consolidator grant. The University of Nottingham and the HPC Midlands Plus Centre are gratefully acknowledged for providing computational resources.

\section{Conflicts of interest}

There are no conflicts of interest to declare.

\section{References}

1 D. P. Goronzy, M. Ebrahimi, F. Rosei, Arramel, Y. Fang, S. De Feyter, S. L. Tait, C. Wang, P. H. Beton, A. T. S. Wee, P. S. Weiss and D. F. Perepichka, ACS Nano, 2018, DOI: 10.1021/acsnano.8b03513.

2 K. S. Mali, N. Pearce, S. De Feyter and N. R. Champness, Chem. Soc. Rev., 2017, 46, 2520-2542.

3 C. W. Tang, Appl. Phys. Lett., 1986, 48, 183-185.

4 Z. Bao, A. Lovinger and A. Dodabalapur, Appl. Phys. Lett., 1996, 69, 3066-3068.
5 J. Puigdollers, C. Voz, M. Fonrodona, S. Cheylan, M. Stella, J. Andreu, M. Vetter and R. Alcubilla, J. Non-Cryst. Solids, 2006, 352, 1778-1782.

$6 \mathrm{~J}$. Nelson, The physics of solar cells, World Scientific Publishing Co. Inc., 2003.

7 A. Fujii, Y. Ohmori and K. Yoshino, IEEE Trans. Electron Devices, 1997, 44, 1204-1207.

8 A. Wilson and J. D. Wright, Mol. Cryst. Liq. Cryst., 1992, 211, 321-326.

9 G. de la Torre, C. G. Claessens and T. Torres, Chem. Commun., 2007, 2000-2015.

10 B. Doppagne, M. C. Chong, H. Bulou, A. Boeglin, F. Scheurer and G. Schull, Science, 2018, 361, 251-255.

11 H. Imada, K. Miwa, M. Imai-Imada, S. Kawahara, K. Kimura and Y. Kim, Nature, 2016, 538, 364-367.

12 Y. Zhang, Y. Luo, Y. Zhang, Y.-J. Yu, Y.-M. Kuang, L. Zhang, Q.-S. Meng, Y. Luo, J.-L. Yang, Z.-C. Dong and J. G. Hou, Nature, 2016, 531, 623-627.

13 V. V. Korolkov, M. Baldoni, K. Watanabe, T. Taniguchi, E. Besley and P. H. Beton, Nat. Chem., 2017, 9, 1191-1197.

14 V. V. Korolkov, S. A. Svatek, A. Summerfield, J. Kerfoot, L. Yang, T. Taniguchi, K. Watanabe, N. R. Champness, N. A. Besley and P. H. Beton, ACS Nano, 2015, 9, 10347-10355.

15 J. Kerfoot, V. V. Korolkov, A. S. Nizovtsev, R. Jones, T. Taniguchi, K. Watanabe, I. Lesanovsky, B. Olmos, N. A. Besley, E. Besley and P. H. Beton, J. Chem. Phys., 2018, 149, 054701.

16 H. Yanagi, T. Kouzeki, M. Ashida, T. Noguchi, A. Manivannan, K. Hashimoto and A. Fujishima, J. Appl. Phys., 1992, 71, 5146-5153.

17 X. Lu, K. W. Hipps, X. D. Wang and U. Mazur, J. Am. Chem. Soc., 1996, 118, 7197-7202.

18 M. D. Upward, P. H. Beton and P. Moriarty, Surf. Sci., 1999, 441, 21-25. 19 S. K. Hämäläinen, M. Stepanova, R. Drost, P. Liljeroth, J. Lahtinen and J. Sainio, J. Phys. Chem. C, 2012, 116, 20433-20437.

20 J.-D. Chai and M. Head-Gordon, Phys. Chem. Chem. Phys., 2008, 10, 6615.

21 T. H. Dunning, J. Chem. Phys., 1989, 90, 1007-1023.

22 Y. Shao, Z. Gan, E. Epifanovsky, A. T. B. Gilbert, M. Wormit, J. Kussmann, A. W. Lange, A. Behn, J. Deng, X. Feng, D. Ghosh, M. Goldey, P. R. Horn, L. D. Jacobson, I. Kaliman, R. Z. Khaliullin, T. Kus, A. Landau, J. Liu, E. I. Proynov, Y. M. Rhee, R. M. Richard, M. A. Rohrdanz, R. P. Steele, E. J. Sundstrom, H. L. Woodcock, P. M. Zimmerman, D. Zuev, B. Albrecht, E. Alguire, B. Austin, G. J. O. Beran, Y. A. Bernard, E. Berquist, K. Brandhorst, K. B. Bravaya, S. T. Brown, D. Casanova, C. M. Chang, Y. Chen, S. H. Chien, K. D. Closser, D. L. Crittenden, M. Diedenhofen, R. A. Distasio, H. Do, A. D. Dutoi, R. G. Edgar, S. Fatehi, L. Fusti-Molnar, A. Ghysels, A. GolubevaZadorozhnaya, J. Gomes, M. W. D. Hanson-Heine, P. H. P. Harbach, A. W. Hauser, E. G. Hohenstein, Z. C. Holden, T. C. Jagau, H. Ji, B. Kaduk, K. Khistyaev, J. Kim, J. Kim, R. A. King, P. Klunzinger, D. Kosenkov, T. Kowalczyk, C. M. Krauter, K. U. Lao, A. D. Laurent, K. V. Lawler, S. V. Levchenko, C. Y. Lin, F. Liu, E. Livshits, R. C. Lochan, A. Luenser, P. Manohar, S. F. Manzer, S. P. Mao, N. Mardirossian, A. V. Marenich, S. A. Maurer, N. J. Mayhall, E. Neuscamman, C. M. Oana, R. OlivaresAmaya, D. P. Oneill, J. A. Parkhill, T. M. Perrine, R. Peverati, A. Prociuk, D. R. Rehn, E. Rosta, N. J. Russ, S. M. Sharada, S. Sharma, D. W. Small, A. Sodt, T. Stein, D. Stück, Y. C. Su, A. J. W. Thom, T. Tsuchimochi, V. Vanovschi, L. Vogt, O. Vydrov, T. Wang, M. A. Watson, J. Wenzel, A. White, C. F. Williams, J. Yang, S. Yeganeh, S. R. Yost, Z. Q. You, I. Y. Zhang, X. Zhang, Y. Zhao, B. R. Brooks, G. K. L. Chan, D. M. Chipman, C. J. Cramer, W. A. Goddard, M. S. Gordon, W. J. Hehre, A. Klamt, H. F. Schaefer, M. W. Schmidt, C. D. Sherrill, D. G. Truhlar, A. Warshel, X. Xu, A. Aspuru-Guzik, R. Baer, A. T. Bell, N. A. Besley, J. Da Chai, A. Dreuw, B. D. Dunietz, T. R. Furlani, S. R. Gwaltney, C. P. Hsu, Y. Jung, J. Kong, D. S. Lambrecht, W. Liang, C. Ochsenfeld, V. A. Rassolov, L. V. Slipchenko, J. E. Subotnik, T. Van Voorhis, J. M. Herbert, A. I. Krylov, P. M. W. Gill and M. Head-Gordon, Mol. Phys., 2015, 113, 184-215.

23 C. Murray, N. Dozova, J. G. McCaffrey, N. Shafizadeh, W. Chin, M. Broquier and C. Crépin, Phys. Chem. Chem. Phys., 2011, 13, 17543.

24 M. Müller, A. Paulheim, A. Eisfeld and M. Sokolowski, J. Chem. Phys., 2013, 139, 044302.

25 A. Eisfeld, C. Marquardt, A. Paulheim and M. Sokolowski, Phys. Rev. Lett., 2017, 119, 097402.

26 R. Forker, T. Dienel, A. Krause, M. Gruenewald, M. Meissner, T. Kirchhuebel, O. Gröning and T. Fritz, Phys. Rev. B: Condens. Matter Mater. Phys., 2016, 93, 165426.

27 B. P. Krueger, G. D. Scholes and G. R. Fleming, J. Phys. Chem. B, 1998, 102, 5378-5386. 